\title{
Resting metabolic rate and the menstrual cycle
}

\author{
M. Campolier, S. Thondre and H. Lightowler \\ Functional Food Centre, Department of Sport and Health Sciences, Oxford Brookes University, Gipsy Lane, \\ Headington, Oxford, OX3 OBP
}

Previous research has shown a biphasic pattern underlying resting metabolic rate (RMR) in women, namely RMR greater in the luteal phase (LP) than in the follicular phase (FP) of the menstrual cycle (MC) ${ }^{(1,2)}$, although others have shown no differences between phases ${ }^{(3,4)}$. When alterations have been found, these have been attributed to changes in ovarian hormones, i.e. oestrogen and progesterone, throughout the MC. The aim of this study was to investigate whether RMR fluctuates significantly in women with regular and natural MC.

Eleven healthy women, aged 26.6 (SD 5.9) y, BMI 22.7 (SD 2.2) kg/m² and experiencing regular MC (25-35 days), were tested three times a week during an entire MC. Subjects attended the laboratory after an overnight fast for the assessment of their RMR, oestrogen and progesterone levels. RMR was measured for $30 \mathrm{~min}$ by indirect calorimetry using a ventilated hood system. Ovarian hormone levels were measured in venous blood sample and analysed by an Electrochemiluminescense Immuno-Assay (ECLIA). RMR fluctuations were determined by co-efficient of variation $(\mathrm{CV} \%)$ and differences between phases were analysed using one-way ANOVA for repeated measures.

There were no significant differences in RMR between the menstrual phase (MP), FP or LP. Mean intra-individual variation (CV\%) in RMR was 6.3 (SD 2.8) \% and ranged from 3.0-11.7\%. When dividing the sample between women with high $(n 5)$ and low $(n 6)$ intra-individual variation ( $\mathrm{CV}>$ and $\leq 5 \%$, respectively) the difference in RMR between the LP and FP increased in the high CV group, although it did not reach significance.

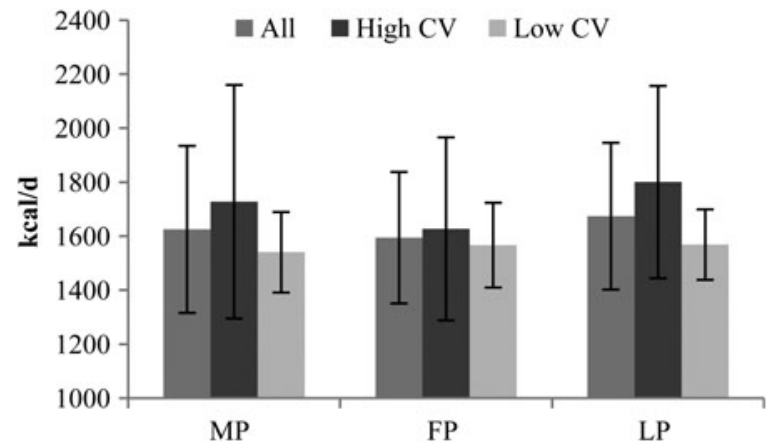

\begin{tabular}{|c|c|c|c|c|c|c|}
\hline & \multicolumn{2}{|l|}{ MP } & \multicolumn{2}{|l|}{$\mathrm{FP}^{*}$} & \multicolumn{2}{|l|}{$\mathrm{LP}^{\S}$} \\
\hline & $\overline{\text { Mean }}$ & SD & Mean & SD & Mean & SD \\
\hline Oestrogen $(\mathrm{pg} / \mathrm{ml})$ & $45 \cdot 46$ & $19 \cdot 58^{* \&}$ & 174.73 & $68 \cdot 02$ & $178 \cdot 28$ & $42 \cdot 93$ \\
\hline Progesterone $(\mathrm{ng} / \mathrm{ml})$ & 0.849 & $0 \cdot 169^{*} \S$ & 0.826 & $0 \cdot 269^{\S}$ & $11 \cdot 342$ & $3 \cdot 327$ \\
\hline
\end{tabular}

Values are means of hormones for three phases. ${ }^{*}$ Significantly different to the

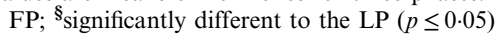

The LP presented significantly higher oestrogen and progesterone levels than the MP and higher progesterone than the FP. Moreover, oestrogen was significantly greater in the FP than the MP. There were no correlations between the hormones and the $\mathrm{RMR}$ in the different phases. There were no significant differences in hormone levels throughout the MC between the high and low $\mathrm{CV} \%$ groups.

Although no significant differences were found in RMR between the phases of the MC in all subjects, there was a trend towards a higher RMR in the LP and this was particularly evident in females with a high CV\% in their RMR. Further work is required to investigate the exact mechanisms underlying these variations.

1. Solomon SJ, Kurzer MS \& Calloway DH (1982). Am J Clin Nutr 36, 611-6.

2. Howe JC, Rumpler WV \& Seale JL (1993). J Nutr Biochem 4, 268-73.

3. Piers LS, Diggavi SN, Rijskamp J, et al. (1995) Am J Clin Nutr 61, 296-302.

4. Henry CJK, Lightowler HJ \& Marchini J (2003). Br J Nutr 89, 811-817. 